\title{
Rhabdomyolysis after High Intensity Resistance Training
}

\author{
Sakiko Honda ${ }^{1}$, Tatsuya Kawasaki ${ }^{1}$, Tadaaki Kamitani ${ }^{1}$ and Keisuke Kiyota ${ }^{2}$
}

\begin{abstract}
Rhabdomyolysis, which is a characteristic occurrence in associated with muscle cell necrosis, develops due to various causes. We herein report a rare case of a patient with rhabdomyolysis after high intensity resistance training, in which markedly elevated levels of serum creatine kinase (CK) and urine myoglobin were observed. A previously healthy 37 -year-old man presented with severe myalgia and dark urine after performing high-intensity exercise. The patient's serum CK level was 95,100 U/L and his urine myoglobin level was $160,000 \mathrm{ng} / \mathrm{mL}$. His symptoms and laboratory findings gradually improved with the intravenous administration of saline and no complications (including electrolyte imbalance and acute renal failure) developed.
\end{abstract}

Key words: creatine kinase, exercise, myoglobin, rhabdomyolysis, scintigraphy

(Intern Med 56: 1175-1178, 2017)

(DOI: 10.2169/internalmedicine.56.7636)

\section{Introduction}

Rhabdomyolysis is a serious condition that is characterized by the necrosis of muscle cells and which can be provoked by a variety of causes, such as trauma, drug toxicity (e.g., statins and fibrates), prolonged immobilization, and infection (1-4). We herein report a rare case of rhabdomyolysis after high intensity resistance training, in a patient with extremely elevated levels of serum creatine kinase (CK) and urine myoglobin.

\section{Case Report}

A 37-year-old man was admitted to Matsushita Memorial Hospital due to myalgia and dark urine. The patient had been well five days prior to his admission, when myalgia developed in the chest and both upper limbs after intense exercise training that included 100 pushups, 100 exercises using a 20-kg dumbbell, 50 lifts using a 10-kg weight. The resistance training was reportedly performed in a gym with a new trainer. He stated that he had exercised regularly but had never performed such intense training before. The next day, he noticed dark urine. The muscle pain deteriorated and he was forced to spend a few days resting in bed. The myalgia was gradually alleviated, but the dark urine continued. He visited his physician four days after the intense exercise and was found to have a serum CK of 65,900 U/L. His previous medical history was unremarkable. The patient did not smoke, drink alcohol, or use illicit drugs and had no known allergies. He was taking loxoprofen sodium, as needed, for toothache. He had no history of statin use.

A physical examination revealed that the patient was 170 $\mathrm{cm}$ tall and weighed $89 \mathrm{~kg}$ (body mass index, 30.8), with a blood pressure of $150 / 109 \mathrm{mmHg}$, pulse of 84 beats per minute, body temperature of $36.9^{\circ} \mathrm{C}$, and an oxygen saturation of $98 \%$ on room air. Muscle tenderness was observed in the chest and upper limbs, with no specific change in the appearance of the skin of the affected areas, such as swelling or redness. Although a manual muscle test was not performed due to severe myalgia, a grade of $3 / 5$ or more was expected. The other examination results were normal. An electrocardiogram showed sinus rhythm, right axis deviation, and flat $\mathrm{T}$ waves in leads V5 and V6. A chest radiograph was normal. The patient's level of serum CK was 95,100 U/ $\mathrm{L}$ with an $\mathrm{MB}$ fraction of $0.3 \%$ (reference value, $\leq 6$ ). As shown in Table, the levels of lactate dehydrogenase, aspartate transaminase, and alanine aminotransferase were elevated to 4,750 U/L, $999 \mathrm{U} / \mathrm{L}$, and $443 \mathrm{U} / \mathrm{L}$, respectively. The patient's renal function was normal, as were his complete blood cell counts and electrolyte levels. A urinalysis revealed light brown urine (Fig. 1), with $3^{+}$occult blood, $2^{+}$ protein, a specific gravity of 1.026 , a $\mathrm{pH}$ of 6.0 , and a myoglobin level of $160,000 \mathrm{ng} / \mathrm{mL}$ (reference value, $\leq 10$ ).

${ }^{1}$ Department of Cardiology, Matsushita Memorial Hospital, Japan and ${ }^{2}$ Kiyota Clinic, Japan

Received for publication April 26, 2016; Accepted for publication September 14, 2016

Correspondence to Dr. Sakiko Honda, pitika325@gmail.com 
Table. The Laboratory Data.

\begin{tabular}{lrrrrrr}
\hline Variable & Reference value & On admission & Day 2 & Day 4 & Day 7 & Day 9 \\
\hline Creatine kinase (U/L) & $\leq 250$ & 95,100 & 58,230 & 20,304 & 2.689 & 944 \\
Lactate dehydrogenase (U/L) & $110-220$ & 4,750 & 2,134 & 617 & 262 & 274 \\
Aspartate aminotransferase (U/L) & $\leq 35$ & 999 & 753 & 469 & 124 & 90 \\
Alanine aminotransferase (U/L) & $\leq 40$ & 443 & 358 & 375 & 239 & 212 \\
C-reactive protein (mg/dL) & $\leq 0.25$ & 1.90 & 1.55 & 0.70 & 0.38 & 0.27 \\
Sodium (mmol/L) & $135-147$ & 142 & 139 & 142 & 142 & 142 \\
Potassium (mmol/L) & $3.6-5.0$ & 4.2 & 4.3 & 4.1 & 3.8 & 3.8 \\
Chloride (mmol/L) & $100-110$ & 105 & 105 & 107 & 106 & 107 \\
Calcium (mg/dL) & $8.2-9.8$ & 9.4 & 8.7 & 9.0 & 9.0 & 9.2 \\
Urea nitrogen (mg/dL) & $8-20$ & 15 & 13 & 9 & 13 & 13 \\
Creatine (mg/dL) & $0.66-1.07$ & 1.05 & 1.01 & 1.02 & 1.03 & 1.06 \\
eGFR (mL/min/1.73m $\left.{ }^{2}\right)$ & $\geq 60$ & 65.2 & 68.1 & 67.3 & 66.6 & 64.6 \\
\hline
\end{tabular}

eGFR: estimated glomerular filtration rate

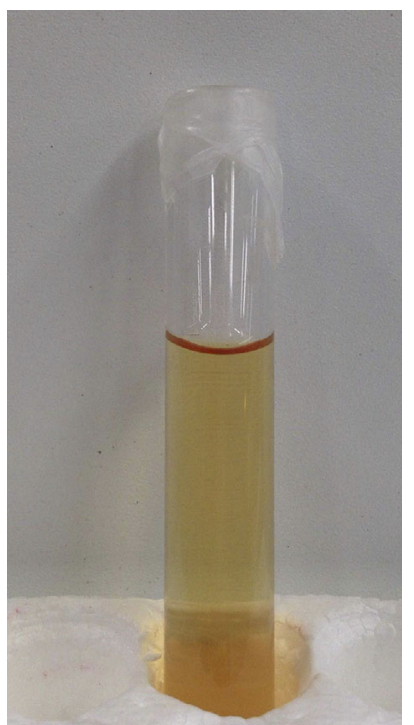

Figure 1. The color of the urine was brown but not dark.

A diagnosis of exertional rhabdomyolysis was made and the intravenous administration of saline was initiated. Scintigraphy with Tc-99m pyrophosphate, performed the day after admission, showed the increased uptake of the radioisotope in the pectoralis major muscle and the biceps and triceps brachii muscles on both sides (Fig. 2). The myalgia gradually improved with a steady decrease in the serum level of CK. No renal dysfunction or electrolyte imbalance was observed (Table). The patient was discharged on the 9th day of hospitalization and he was advised to avoid intense exercise. A follow-up examination at two weeks after discharge revealed that the patient's serum level of CK was still slightly elevated to $280 \mathrm{U} / \mathrm{L}$, possibly due to moderate muscle training. The patient's thyroid function, which was assessed after discharge, was normal with a free T4 level of $1.3 \mathrm{ng} / \mathrm{dL}$ (reference value, 0.9-1.7) and a thyroidstimulating hormone level of $3.01 \mathrm{mIU} / \mathrm{L}$ (reference value, $0.50-5.00)$. The patient was later lost to follow up.

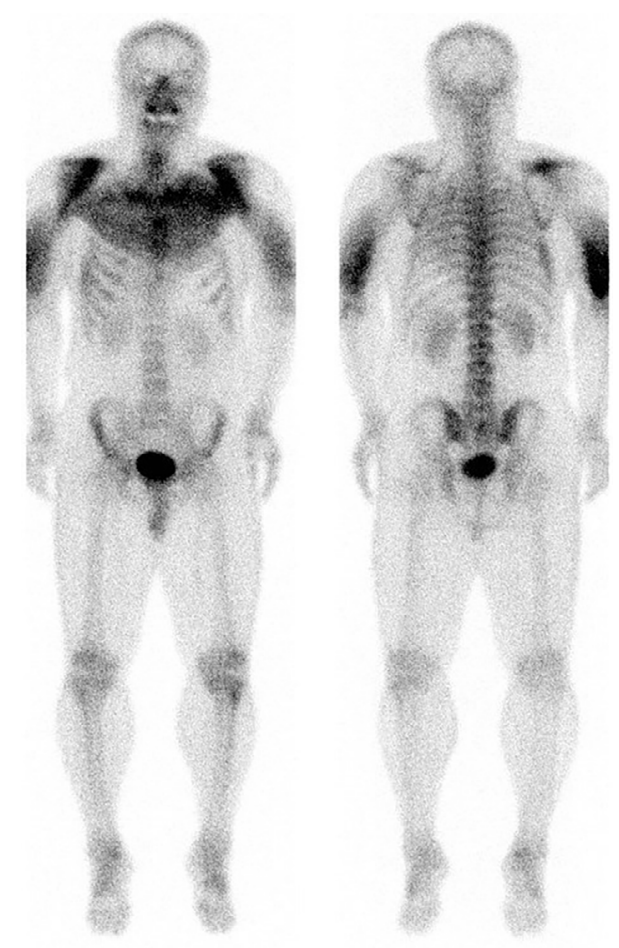

Figure 2. An anterior Tc-99m pyrophosphate scintigraphy image (left panel) showing the increased uptake in the pectoralis major muscle and the right and left biceps brachii muscles. The uptake of the radioisotope was found to be increased in the triceps brachii muscles of both sides on the posterior image (right panel).

\section{Discussion}

The triad of symptoms associated with rhabdomyolysis includes myalgia, muscle weakness, and dark urine. Less than $10 \%$ of the affected patients have all three symptoms (1). In the current case, the patient's myalgia was severe but muscle weakness was not obvious. The urine color on admission was not typical; however, the level of urine myoglobin was markedly elevated. The color of urine can be 
affected by many factors including the muscle mass, urine concentration, and glomerular function (1). It is reported that only $3.6 \%$ of patients with rhabdomyolysis (defined by serum CK levels of $>1,000 \mathrm{U} / \mathrm{L}$ ) had a history of dark urine (5). Furthermore, more than half of rhabdomyolysis patients do not exhibit muscle pain or weakness (1). Taken together, this suggests that the physicians' suspicion (based on the patient's history), followed by laboratory confirmation (serum CK levels of $>5$ times the upper limit of the normal reference value or 1,000 U/L), is crucial for diagnosing rhabdomyolysis $(1,6)$. As shown in our case, scintigraphy with Tc-99m pyrophosphate can be used to assess the degree and extent of muscle damage $(7,8)$.

Given that serum $\mathrm{CK}$ is the most common marker for assessing the degree of muscle damage, it is reasonable that serum CK levels would increase after exercise according to the intensity of the exercise (9). In a study of 15 marathon runners who completed the official distance of $42.195 \mathrm{~km}$, the levels of serum CK increased from $161 \mathrm{U} / \mathrm{L}$ before the race to $3,424 \mathrm{U} / \mathrm{L}$ at 24 hours after finishing the race (10). In addition, the increased $\mathrm{CK}$ levels were significantly higher in the runners who finished the race within 210 minutes than it was in runners who finished the race at over 210 minutes (on average, 4,433 U/L versus 1,432 U/L, respectively). In an analysis of 36 runners who completed a 246$\mathrm{km}$ ultramarathon race within the 36-hour time limit (11), the blood samples obtained within 15 minutes after the end of the race showed a mean serum CK of 43,762 U/L; however, no significant correlation was observed between the finishing times and the serum CK levels.

The incidence of exertional rhabdomyolysis remains unclear, probably because exercise varies in intensity and individual tolerance. A study of military recruits who performed basic training revealed that 22.2 cases of exertional rhabdomyolysis occurred per 100,000 individuals per year (approximately $0.022 \%$; among 198,399 recruits) (12). It should also be noted that the recurrence of rhabdomyolysis in the same cohort was only observed in one case during the mean follow-up period of 31.2 months. This may be explained by the fact that the onset of exertional rhabdomyolysis is affected not only by the exercise intensity but also by environmental conditions, including the temperature and humidity during exercise (13).

Two mechanisms have been proposed to underlie the development of exertional rhabdomyolysis: muscle membrane injury and energy depletion (14). Given the relatively short period of high intensity resistance exercise in our case, muscle membrane injury is likely to have been the main cause of the patient's rhabdomyolysis. However, it remains controversial whether the intensity of the exercise was consistent with the markedly elevated levels of serum CK. It is also noted that the patient's serum CK levels peaked at five days after the high intensity resistance training, despite the fact that the half-life of serum CK is 1.5 days (1). These findings might suggest the presence of other compounding factors, such as muscle ischemia due to muscle swelling, im- mobilization due to severe muscle pain, and dehydration due to prolonged immobilization. It has been reported that breaching the integrity of the muscle membrane can lead to a constellation of physiological changes: namely, an inflammatory, self-sustaining myolysis cascade $(1,14-17)$.

Among the potential complications associated with rhabdomyolysis, acute renal failure, compartment syndrome, and disseminated intravascular coagulation can be lifethreatening (18). Acute renal injury is estimated to occur in approximately one third of rhabdomyolysis patients (18), whereas the incidence of the other two complications remains unknown but seems less frequent. Direct injury to the kidneys by the accumulation of myoglobin is considered to be the main cause of the acute renal failure associated with rhabdomyolysis $(1,18)$. It is reasonable that higher serum levels of $\mathrm{CK}$, such as $>5,000 \mathrm{U} / \mathrm{L}$, would be associated with the development of acute renal failure (1). When acute renal failure is suspected in rhabdomyolysis patients, aggressive fluid replacement as well as dialysis should immediately be considered $(1,6,19)$. In our case, no serious complications occurred, however, there was a marked elevation in the patient's serum level of CK and urine level of myoglobin. The rate of acute renal failure among exertional rhabdomyolysis patients has been reported to be lower than that among nonexertional rhabdomyolysis patients (13).

\section{The authors state that they have no Conflict of Interest (COI).}

\section{Acknowledgement}

We thank Dr. Hiroki Sugihara for his thoughtful comments on the manuscript.

\section{References}

1. Torres PA, Helmstetter JA, Kaye AM, Kaye AD. Rhabdomyolysis: pathogenesis, diagnosis, and treatment. Ochsner J 15: 58-69, 2015.

2. Melli G, Chaudhry V, Cornblath DR. Rhabdomyolysis: an evaluation of 475 hospitalized patients. Medicine (Baltimore) 84: 377385, 2005.

3. Watanabe T. Rhabdomyolysis and acute renal failure in children. Pediatr Nephrol 16: 1072-1075, 2001.

4. Watemberg N, Leshner RL, Armstrong BA, Lerman-Sagie $\mathrm{T}$. Acute pediatric rhabdomyolysis. J Child Neurol 15: 222-227, 2000 .

5. Mannix R, Tan ML, Wright R, Baskin M. Acute pediatric rhabdomyolysis: causes and rates of renal failure. Pediatrics 118: 21192125, 2006.

6. Cervellin G, Comelli I, Lippi G. Rhabdomyolysis: historical background, clinical, diagnostic and therapeutic features. Clin Chem Lab Med 48: 749-756, 2010.

7. Ludmer LM, Chandeysson P, Barth WF. Diphosphonate bone scan in an unusual case of rhabdomyolysis: a report and literature review. J Rheumatol 20: 382-384, 1993.

8. Cornelius EA. Nuclear medicine imaging in rhabdomyolysis. Clin Nucl Med 7: 462-464, 1982.

9. Stansbie D, Aston JP, Dallimore NS, Williams HM, Willis N. Effect of exercise on plasma pyruvate kinase and creatine kinase activity. Clin Chim Acta 132: 127-132, 1983.

10. Siegel AJ, Silverman LM, Lopez RE. Creatine kinase elevations in marathon runners: relationship to training and competition. Yale $\mathrm{J}$ Biol Med 53: 275-279, 1980. 
11. Skenderi KP, Kavouras SA, Anastasiou CA, Yiannakouris N, Matalas AL. Exertional Rhabdomyolysis during a 246-km continuous running race. Med Sci Sports Exerc 38: 1054-1057, 2006.

12. Alpers JP, Jones LK Jr. Natural history of exertional rhabdomyolysis: a population-based analysis. Muscle Nerve 42: 487-491, 2010.

13. Schwaber MJ, Liss HP, Steiner I, Brezis M. Hazard of sauna use after strenuous exercise. Ann Intern Med 120: 441-442, 1994.

14. Milne CJ. Rhabdomyolysis, myoglobinuria and exercise. Sports Med 6: 93-106, 1988.

15. Al-Ismaili Z, Piccioni M, Zappitelli M. Rhabdomyolysis: pathogenesis of renal injury and management. Pediatr Nephrol 26: 1781-1788, 2011

16. Huerta-Alardín AL, Varon J, Marik PE. Bench-to-bedside review: rhabdomyolysis - an overview for clinicians. Crit Care 9: 158-169, 2005.

17. Vanholder R, Sever MS, Erek E, Lameire N. Rhabdomyolysis. J Am Soc Nephrol 11: 1553-1561, 2000.

18. Khan FY. Rhabdomyolysis: a review of the literature. Neth J Med 67: 272-283, 2009.

19. Elsayed EF, Reilly RF. Rhabdomyolysis: a review, with emphasis on the pediatric population. Pediatr Nephrol 25: 7-18, 2010.

The Internal Medicine is an Open Access article distributed under the Creative Commons Attribution-NonCommercial-NoDerivatives 4.0 International License. To view the details of this license, please visit (https://creativecommons.org/licenses/ by-nc-nd/4.0/).

(C) 2017 The Japanese Society of Internal Medicine http://www.naika.or.jp/imonline/index.html 